\title{
The Greater Value of the CONSORT Statement Guidelines: Guideposts for Designing and Reporting all TMB Research
}

\author{
Antony J. Porcino, BSc, $\mathrm{PhD}$ \\ Complementary Medicine Education and Outcomes Research Program, British Columbia Cancer Agency, \\ Vancouver, BC, Canada
}

The IJTMB recommends the use of the CONSORT Statement (Consolidated Standards of Reporting Trials) guidelines for the reporting of randomized, controlled clinical trials (RCTs). A careful review of the guidelines shows important applications of these guidelines to all types of research reporting and design, not just RCTs. There is an Extension to these guidelines specific to nonpharmacologic interventions, including manually applied therapies and complementary medicine, and thus therapeutic massage and bodywork (TMB). Components of the Extension are thus relevant to publication in the IJTMB and should be considered part of standard reporting. As well, while the goals of the CONSORT Statement guidelines are to improve reporting of RCTs, the issues raised in the guidelines and explanatory document are relevant to all forms of TMB research and should be considered in all TMB research manuscripts. Finally, while not their purpose, the guidelines could also be used as an informal checklist when developing robust TMB research.

In its Author Guidelines, the IJTMB advises the use of the CONSORT Statement (Consolidated Standards of Reporting Trials) and PRISMA guidelines (Preferred Reporting Items for Systematic Reviews and Meta-Analyses, replacing QUORUM in 2009). These guidelines were developed by international panels to help create a consistent presentation of important information when publishing research results $^{(1)}$. The application of such guidelines serves both the researchers and the readership, by reminding authors of what information is valuable to readers not involved in the research, and providing a degree of certainty for readers that the requisite guideposts will be in place for properly assessing, and possibly using, the research.

The CONSORT Statement was originally developed to address problems that arose from inadequate and inconsistent reporting of randomized controlled trial (RCT) details ${ }^{(1)}$. However, the CONSORT Group specifically recognizes that in nonpharmacological treatments, certain issues provide challenges not well-addressed in the CONSORT Statement, including complex interventions, treatment provider expertise, and difficulties with blinding ${ }^{(2)}$. While there are several reasons why this may be the case, many of which are addressed within the more specialized CONSORT Extension for Non-Pharmaceutical Interventions, of particular interest to the therapeutic massage and bodywork (TMB) field are the issues arising from the necessary additional complexities of TMB research interventions relative to classic pharmaceutical $\mathrm{RCTs}^{(2)}$. For example, while a placebo is rarely appropriate or possible, and blinding of both/ either providers and recipients to the treatment can be difficult, the alternatives such as treatment shams, waitlist, standard care comparisons, multiarm trials, and comparison effectiveness trials often lead to more complex research designs ${ }^{(3,4)}$; as well, practitioner expertise affects research outcomes ${ }^{(5,6)}$. The Extension recommendations address some key issues regarding these problems, particularly on how to report them in the manuscript. This includes reporting precise details of both the treatment and the comparison treatment; providing descriptions of how procedures are adapted to individual patients; detailing how interventions were standardized if multiple practitioners provide the treatments, including quality control processes; and recommendations on discussing how these issues relate to the generalizability of the results. By reporting on these details, authors would further increase the quality and consistency of IJTMB articles.

As part of a team, I have just submitted a systematic review of complementary medicine use in advanced cancer care, for consideration of publication. One of the surprising things we found in doing a mixed studies review-including quantitative, qualitative, mixed methods research articles, and systematic reviews - was how relatively few research articles adequately publish basic information about their methods. This omission limited our ability to adequately assess the quality of the methods applied in the research, and limited the external validity of the work we were reviewing. This implies that choosing to employ the CONSORT Statement guidelines, and (when appropriate) extensions, to all forms of research would likely result in systematic reviews with greater strength to their conclusions - something to 
think about as TMB research develops a large enough research base to make TMB systematic reviews or meta-analyses practical.

As Executive Editor, researcher, and research consumer, I welcome the application of the CONSORT Statement guidelines, and specifically the Extension for nonpharmacological interventions, to therapeutic massage and bodywork research manuscripts. Readers like to see precise details of the chosen research methods, allowing the reasoning of the design choices to be understood. Additionally, because TMB practitioners have highly variable skill sets, experience ${ }^{(7,8)}$, and likely differing bedside manners, readers like to understand how these were dealt with to minimize impact on the research outcomes. Similarly, how patient variables such as expectation effects and experiences with the therapy are addressed, are rarely described, but should be, a common oversight in research generally. These issues also extend beyond manuscript writing. Manuscript guidelines are never meant as research design guidelines because they cannot address all issues of research design and implementation. Even so, the issues raised in the CONSORT Extension guidelines should be carefully reviewed when designing research to ensure that the complex issues affecting TMB research are considered throughout the development of the research plan, right from its conception. The strength of including review of manuscript guidelines during the research design process was suggested as early as $1991^{(9)}$ and this recommendation remains as relevant today.

While the CONSORT Statement guidelines were developed for improving the reporting of RCTs, I believe that many of the issues discussed in the guidelines and the nonpharmaceutical Extension are relevant to all forms of research in the TMB field. For example, efforts to isolate and clarify treatment effect must also be undertaken in longitudinal and casecontrolled studies, and even single-subject research and case reports. Similarly, therapist and patient variability is an issue in all research, and therefore should be addressed in all research manuscripts and, hopefully, in research design.

Therefore, I recommend that all authors of all research consider the underlying purpose guiding the development of the CONSORT Statement guidelines and the nonpharmacologic Extension, and review them for both research design and manuscript writing for any research undertaken, right from the start, to ensure that the appropriate issues have been considered and adequately documented. Additionally, when manuscripts of the research are prepared, researchers will have the necessary information readily available. I will start by stating that I am still learning to do so, but that these principles and related knowledge translation processes are being built into my current research projects, from the ground up. I hope other researchers will consider following this practice as well.

\section{COPYRIGHT}

Published under the CreativeCommons AttributionNonCommercial-NoDerivs 3.0 License.

\section{REFERENCES}

1. Altman D, Schulz K, Moher D, et al. The revised CONSORT statement for reporting randomized trials: explanation and elaboration. Ann Intern Med. 2001;134(8):663-694.

2. Boutron I, Moher D, Altman D, et al. Extending the CONSORT statement to randomized trials of nonpharmacologic treatment: explanation and elaboration. Ann Intern Med. 2008;148(4):295-309.

3. Boutron I, Tubach F, Giraudeau B, et al. Blinding was judged more difficult to achieve and maintain in nonpharmocologic than pharmacologic trials. $J$ Clin Epidemiol. 2004;57(5):543-550.

4. Mein EA, Greenman PE, McMillin DL, et al. Manual medicine diversity: research pitfalls and the emerging medical paradigm. J Am Osteopath Assoc. 2001;101(8):441-446.

5. Moraska A. Therapist education impacts the massage effect on postrace muscle recovery. Med Sci Sports Exerc. 2007;39(1):34-37.

6. Donoyama N, Shibasaki M. Differences in practitioners' proficiency affect the effectiveness of massage therapy on physical and psychological states. J Bodyw Move Ther. 2010;14(3):239-244.

7. American Massage Therapy Association. 2010 Massage Profession Research Report. Evanstan, ILL AMTA; 2010.

8. Porcino A, Boon H, Page S, et al. Meaning and challenges in the practice of multiple therapeutic massage modalities: a combined methods study. BMC Complement Altern Med. 2011;11:75.

9. Findley TW. Research in physical medicine and rehabilitation. IX. Primary data analysis. Am J Phys Med Rehabil. 1990;69(4):209-218. 